\title{
Risk Assessment for Venous Thromboembolism in Patients With Neuroepithelial Tumors: Pretreatment Score to Identify High Risk Patients
}

\author{
Tomohiro KAWAgUCHI, ${ }^{1}$ Toshihiro KUMABE, ${ }^{1}$ Masayuki KANAMORI, ${ }^{1}$ \\ Ryuta SAITO, ${ }^{1}$ Yoji YAMASHITA, ${ }^{1}$ Yukihiko SONODA, ${ }^{1}$ and Teiji TOMINAGA ${ }^{1}$ \\ ${ }^{1}$ Department of Neurosurgery, Tohoku University Graduate School of Medicine, Sendai, Miyagi
}

\begin{abstract}
The independent risk factors for venous thromboembolism (VTE) were investigated in patients with neuroepithelial tumor to establish a risk score for VTE. Our prospective study enrolled 395 hospitalized cases with neuroepithelial tumors. All cases underwent measurement of serum D-dimer concentration and neurological examination on admission. Serum D-dimer concentration was measured on days 1, 3, and 7 after surgery and weekly during follow up in patients who underwent surgery, and once a week during follow up in patients without surgery. Fourteen clinical parameters were evaluated as indicators of VTE, and among them, age, body-mass index, chemotherapy, radiation therapy, corticosteroid usage, pretreatment serum D-dimer concentration, paresis of the lower extremity (manual muscle test: MMT), performance status, and World Health Organization grade of the tumor achieved statistical significance. Multivariable logistic regression analysis demonstrated age $>65$ years, corticosteroid usage, paresis of the lower extremity, and serum D-dimer concentration over $1.0 \mathrm{mg} / \mathrm{dl}$ were independent factors. Total risk score was defined as the total of the scores for risk factors assigned based on the adjusted odds ratio: pretreatment serum D-dimer concentration over $1.0 \mathrm{mg} / \mathrm{dl}$ (2 points), and age over 65 years old, paresis of the lower extremity of MMT $\leq 2$, and corticosteroid usage (1 point each). Rates of VTE were $2.0 \%$ in the low risk (total score 0 or 1 ), $14.8 \%$ in the intermediate risk (total score 2 or 3 ), and $\mathbf{5 1 . 9 \%}$ in the high risk groups (total score $=4$ or 5 ). This pretreatment risk score for VTE might be useful to identify patients who would benefit from thromboprophylaxis.
\end{abstract}

Key words: venous thromboembolism, glioma, D-dimer, risk categorization

\section{Introduction}

Venous thromboembolism (VTE) remains a complication carrying high risks of morbidity and mortality in patients undergoing surgery or medical treatment. Specific risk factors have been identified in various populations, ${ }^{4,5,19,25,30,32,34)}$ but these risk factors somewhat depend on the clinical conditions, such as cancer or orthopedic surgery, so a generalized risk assessment model might be hard to establish. High incidences of VTE are associated with brain tumors, and the highest incidence with malignant glioma, ${ }^{1,33,36)}$ variously reported as $20-30 \%$ over the course of the disease. ${ }^{12,15,20,27,31,37)}$ Patients with glioma are considered to have specific conditions as follows: glioma carries a high risk disease of VTE, like other cancers; patients with glioma sometimes develop neurological deficits, such as hemiparesis; chemotherapy, radiation therapy, and corticosteroid usage are sometimes included as postoperative treatments, which result in longer hospitalization; and disease progression sometimes reduces the performance status and activity of daily living. Therefore, glioma patients present with different risks of VTE compared to patients with other cancers or medical diseases.

The present study tried to identify the specific risk factors for VTE in patients with neuroepithelial tumor, by scoring of the predisposing risks.

Received July 12, 2012; Accepted August 9, 2012

Author's present address: T. Kumabe, MD, Department of Neurosurgery, Kitasato University School of Medicine, Sagamihara, Kanagawa, Japan. 


\section{Methods}

This study prospectively enrolled patients with neuroepithelial tumors, such as astrocytic tumors, oligodendroglial tumors, ependymal tumors, other neuroepithelial tumors, and neuronal and mixed neuronal-glial tumors. All patients were admitted for treatment, including surgical resection, biopsy, chemotherapy, or radiation therapy at Tohoku University Hospital between January 2007 and July 2011. Numbers of patients and their hospitalizations were 253 and 395, respectively. In this study, the 395 hospitalized cases were analyzed. Some of them have been reported elsewhere. ${ }^{23)}$ The histological diagnosis was based on the new World Health Organization (WHO) classification. ${ }^{26)}$ Patients with metastatic brain tumor, non-neuroepithelial tumor such as meningioma or germ cell tumor, and spinal cord tumor, and patients under the age of 18 years were excluded. Informed consent was obtained from each patient or guardian on admission, and prior to whole body computed tomography (CT) with contrast medium or anticoagulant therapy.

Risk factors were selected from earlier reports as follows ${ }^{4,5,19,25,30,32,34)}$ : Age, sex, history of cerebrovascular events, presence of diabetes mellitus (DM), cancer, obesity (body-mass index: BMI), surgical treatment, chemotherapy, radiation therapy, any types and dosage of corticosteroid usage, tumor histology (WHO grade), paresis of the lower extremity, performance status, and serum D-dimer level on admission and before treatment including surgery, chemotherapy, and radiation therapy. These variables were prospectively recorded in a computerized database for analysis. Imaging findings, including CT, magnetic resonance imaging, and Doppler ultrasonography of the lower extremity, were also recorded.

The patients were treated following the protocol previously reported. ${ }^{23)}$ The serum D-dimer level was quantitatively measured on admission, and at 1,3 , and 7 days after surgical intervention and every week during follow up in patients who underwent surgery, including tumor resection and biopsy, and every week during follow up in patients who did not undergo surgery. Increased serum D-dimer level exceeding $10.0 \mathrm{mg} / \mathrm{dl}$ indicated further examinations for VTE with Doppler ultrasonography or CT with contrast medium of the chest, abdomen, and lower extremities. Both symptomatic and asymptomatic deep vein thrombosis (DVT) in the lower extremities or pulmonary embolism were defined as VTE in this study.

The means and standard deviations (SDs) for each variable were calculated for the VTE and non-VTE groups. Age, BMI, and manual muscle test (MMT) were compared between the 2 groups with the unpaired $t$ test. Performance status and serum D-dimer level were compared between the 2 groups with the Mann-Whitney test. Other parameters were compared between the 2 groups with the Fisher's exact test. Any variable with a $p$ value of $<0.20$ by univariate analysis was considered a potential independent variable and was entered into the multivariate logistic regression analysis. Pearson's correlation coefficients were calculated, and if highly co-linear factors were both significant, the most easily obtained clinical measure was selected. Multivariate logistic analysis was then performed on the significant variables for calculation of the quantitative risk of VTE onset. The $p$ value for selection as an independent variable was set at 0.05 . Statistical analyses were performed using Microsoft Excel and SPSS (IBM Japan, Tokyo).

Point values were assigned to each risk factor based on the relative adjusted odds ratio, rounded to the nearest integer. The total risk score for each patient was calculated as the sum of the point values. The VTE occurrence rate was calculated for each risk score group.

\section{Results}

The 212 male and 183 female patients were aged 18-89 years (median 53 years). The most frequent tumor histology was glioblastoma (WHO grade IV). During the study period, $43(10.9 \%)$ of the 395 patients developed VTE. The clinical characteristics are summarized in Table 1.

The means and SDs or range for each variable are given in Table 2. Univariate analysis found significant differences for 9 parameters: age $(\mathrm{p}<$ $0.0001)$, BMI ( $p=0.141)$, chemotherapy $(p=0.143)$, radiation therapy $(p=0.100)$, corticosteroid usage ( $p<0.01)$, serum D-dimer level ( $p<0.0001)$, paresis of the lower extremity (MMT) $(p<0.001)$, performance status $(p=0.003)$, and WHO grade of the tumor $(\mathrm{p}<0.05)$.

The 9 significant parameters were further analyzed using multivariate logistic analysis to identify significance accounting for all relevant variables. Before performing regression analysis, all parameters were categorized into 2 groups, age (over or under 65 years), BMI (over or under $25 \mathrm{~kg} / \mathrm{m}^{2}$ ), serum D-dimer level (over or under $1.0 \mathrm{mg} / \mathrm{dl}$ ), and MMT (0-2 or 3-5). Pearson's correlation coefficients were calculated. High correlations were seen between radiation therapy and chemotherapy, WHO grade and chemotherapy, performance status and MMT, respectively. Based on them, radiation therapy, 
Table 1 Baseline demographic characteristics of the total study population $(n=395)$

\begin{tabular}{lcc}
\hline \multicolumn{1}{c}{ Characteristic } & Number of cases & $\%$ \\
\hline Age, range (median), years & $18-89(53)$ & \\
Sex & \multicolumn{2}{c}{} \\
female & 183 & 46.3 \\
male & 212 & 53.7 \\
WHO grade IV & 200 & 50.6 \\
glioblastoma & 199 & 50.4 \\
gliosarcoma & 1 & 0.3 \\
WHO grade III & 97 & 24.6 \\
anaplastic astrocytoma & 43 & 10.9 \\
anaplastic oligodendroglioma & 32 & 8.1 \\
anaplastic oligoastrocytoma & 11 & 2.8 \\
anaplastic ganglioglioma & 4 & 1.0 \\
anaplastic ependymoma & 2 & 0.5 \\
gliomatosis cerebri & 3 & 0.8 \\
glioneuronal tumor & 2 & 0.5 \\
WHO grade II & 68 & 17.2 \\
diffuse astrocytoma & 28 & 7.1 \\
oligodendroglioma & 23 & 5.8 \\
oligoastrocytoma & 9 & 2.3 \\
ependymoma & 4 & 1.0 \\
pleomorphic xanthoastrocytoma & 3 & 0.8 \\
glioneuronal tumor & 1 & 0.3 \\
WHO grade I & 23 & 5.8 \\
pilocytic astrocytoma & 13 & 3.3 \\
ganglioglioma & 10 & 2.5 \\
\hline
\end{tabular}

WHO: World Health Organization.
WHO grade, and performance status were excluded. Finally, logistic regression analysis was performed for age, BMI, chemotherapy, corticosteroid usage, MMT, and serum D-dimer level. Among them, age, corticosteroid usage, MMT, and serum D-dimer level were identified as independently significant parameters (Table 3).

Our scoring strategy for each of the risk factors was intended to assign the total score to a specific absolute risk of VTE. According to the relative adjusted odds ratio, age $>65$ years, MMT $\leq 2$, and corticosteroid usage were assigned 1 point, and serum D-dimer level $>1.0 \mathrm{mg} / \mathrm{dl}$ was assigned 2 points, because the odds ratio of serum D-dimer level was as twice as that of age, MMT, and corticosteroid usage (Table 3). Therefore, the total risk score ranged 0 to 5. Patients with higher score had higher occurrence of VTE onset. Figure 1 shows a probable logarithmic relationship between the risk score and the rate of VTE. The patient population was divided into 3 risk categories based on the risk score: low risk (total score 0 or 1), intermediate risk (total score 2 or 3 ), and high risk (total score 4 or 5). The rate of VTE was $2.0 \%$ in the low risk group, and $14.8 \%$ in the intermediate risk group, and much higher at $51.9 \%$ in the high risk group (Fig. 2).

Table 2 Univariate analysis for all parameters examined in patients with or without venous thromboembolism (VTE)

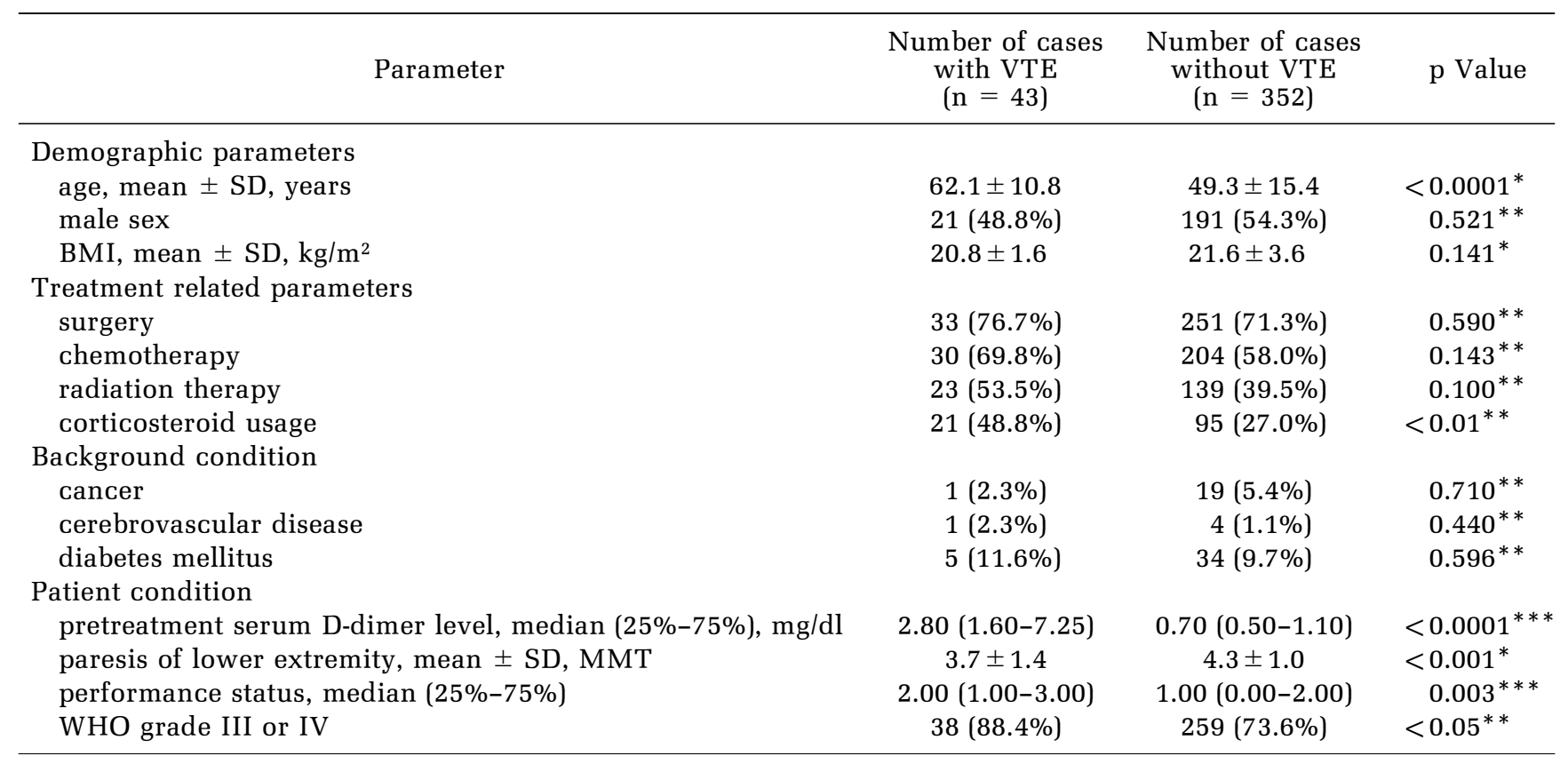

Values determined by *unpaired $t$ test, ${ }^{* *}$ Fisher's exact test, or ${ }^{* * *}$ Mann-Whitney test. BMI: body-mass index, MMT: manual muscle test, SD: standard deviation, WHO: World Health Organization. 
Table 3 Multivariate analysis of venous thromboembolism occurrence in patients with neuroepithelial tumors

\begin{tabular}{lccc}
\hline \multicolumn{1}{c}{ Parameter } & Odds ratio & 95\% Confidence interval & $\mathrm{p}$ Value \\
\hline Age $(>65$ years) & 3.03 & $1.37-6.69$ & 0.006 \\
BMI $\left(>25.0 \mathrm{~kg} / \mathrm{m}^{2}\right)$ & 1.30 & $0.34-5.01$ & 0.70 \\
Chemotherapy & 1.93 & $0.8-4.5$ & 0.12 \\
Corticosteroid usage & 2.12 & $1.03-4.37$ & 0.043 \\
Pretreatment serum D-dimer level $(>1.0 \mathrm{mg} / \mathrm{dl})$ & 5.99 & $2.46-14.57$ & $<0.001$ \\
Paresis of lower extremity (MMT $\leq 2)$ & 2.99 & $1.22-7.30$ & 0.016 \\
\hline
\end{tabular}

BMI: body-mass index, MMT: manual muscle test.

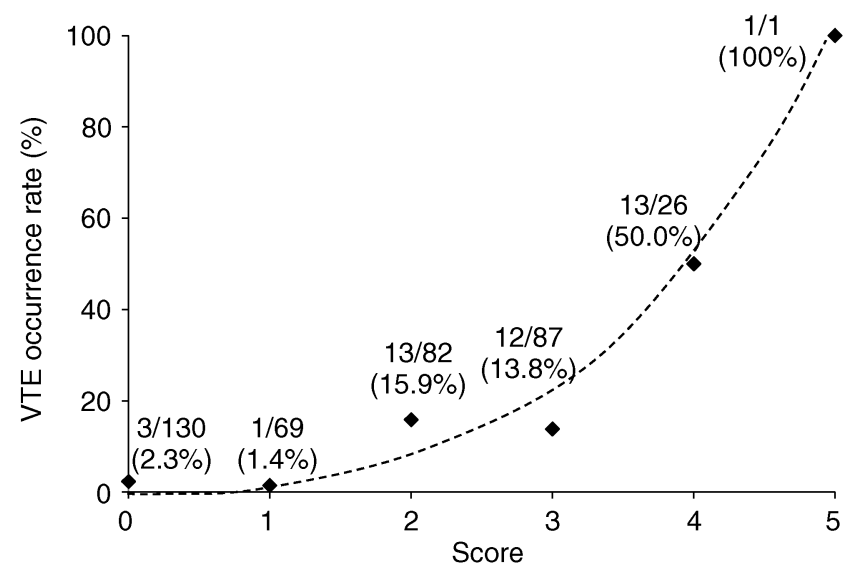

Fig. 1 Risk score plotted against venous thromboembolism (VTE) rate. The graph indicates a logarithmic relationship between risk score and rate of VTE occurrence (number of patients with VTE/total number of patients).

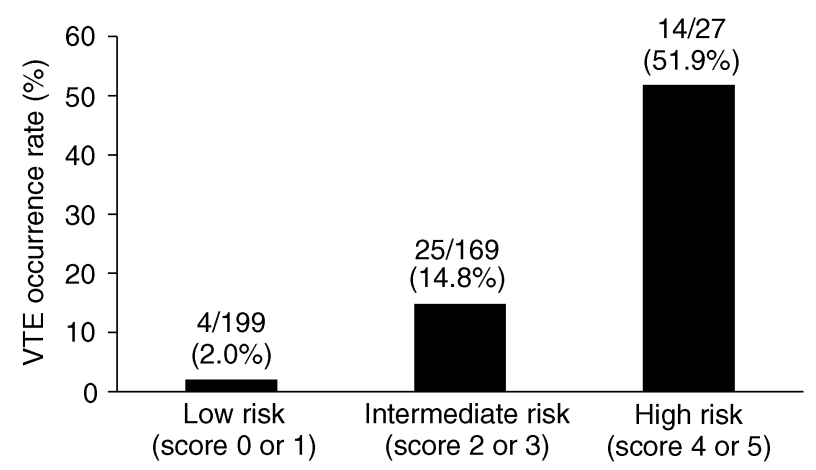

Fig. 2 Three risk groups based on the risk score. Rates of venous thromboembolism (VTE) occurrence were $2.0 \%$ in the low risk (score 0 or 1 ), $14.8 \%$ in the intermediate risk (score 2 or 3 ), and $51.9 \%$ in the high risk groups (score 4 or 5 ).

\section{Discussion}

The present study identified 4 clinical and laborato- ry parameters that were independently associated with the occurrence of VTE: age >65 years, corticosteroid usage, paresis of the lower extremity, and serum D-dimer concentration $>1.0 \mathrm{mg} / \mathrm{dl}$. We propose a scoring strategy for the prediction of VTE based on these parameters, which can always be assessed on admission or before starting treatment, so the scoring strategy is quite simple and easy to introduce in the clinical setting.

All risk factors identified in this study were proposed in earlier studies. ${ }^{21,24,25,33)}$ Corticosteroid usage can be thrombogenic because of the increase in platelet function via activation of factor VIII, ${ }^{28)}$ and can also induce the hypofibrinolytic state. ${ }^{2)}$ Therefore, corticosteroid usage is considered as an independent risk factor for VTE because of these hypercoagulability and hypofibrinolytic states. ${ }^{35)} \mathrm{We}$ could not assess how the dosage or duration of corticosteroid usage affects the VTE occurrence, because of the relatively small sample size. More such cases must be documented and analyzed.

Paresis of the lower extremity is known as a risk factor for VTE. Venous congestion might be occurred, which resulted in DVT. In our series, most cases with DVT had thrombus in the paretic lower extremity. In only one case, DVT was detected in the non-paretic lower extremity. However, the general activity of this patient was low (performance status 4) because of tumor progression. Immobilization of the lower extremity is considered to carry high risk of DVT occurrence.

Serum D-dimer level has a stronger association with VTE onset. D-dimer is the most reliable blood marker for VTE, because the serum concentration immediately increases at the onset of thrombotic events. ${ }^{8-10,38)}$ In fact, serial D-dimer measurement is useful not only for early detection of VTE but also for prediction of VTE occurrence. ${ }^{3,23)}$ Univariate analysis indicated that tumor WHO histology grade had a strong correlation with VTE occurrence ( $p<$ 0.05). However, this factor was intentionally dropped from the risk predictive factor for multivariate analysis because strong co-linear effects 
were observed between WHO grade and age, chemotherapy, performance status, and limb paresis, and because we need to predict the risk of VTE before tumor histology is obtained.

Studies in other settings have shown strong associations between VTE onset and presence of DM, cancer, or cerebrovascular disease, ${ }^{17,18,22,39)}$ which were not recognized as independent risk factors in our study, possibly because the number of patients with these complications was small. In fact, only 5 , 20, and 39 patients had treatment history for cerebrovascular disease, cancer, and DM, respectively.

The present study found the rate of VTE was $10.9 \%$. One previous prospective study found the rate of VTE was $13 \%$ in patients after elective neurosurgery. ${ }^{1)}$ Another prospective study revealed a $20.8 \%$ rate of DVT in glioma patients at 12 months after surgery. ${ }^{7)}$ The duration of follow up was shorter in our study, but our findings are mostly consistent with previous reports. In our study, patients with risk scores of 0 or 1 had a VTE rate of $2.0 \%$. This group was considered to have low risk of VTE, compared to the general occurrence of VTE reported previously. Patients with risk score of 2 or 3 had VTE rate of $14.8 \%$. This group was considered to have intermediate risk of VTE, in the same range as previous reports. ${ }^{23)}$ In contrast, patients with risk score of 4 or 5 had remarkably high VTE occurrence of $51.9 \%$. This group was considered to have high risk of VTE. Although the number of cases was limited, we recommend that intervention should be considered for patients in the high risk group.

VTE is a leading cause of death in patients with various conditions, and the risk of VTE in patients with glioma remains higher than with other malignancies throughout the course of disease. ${ }^{16,21)}$ Therefore, thromboprophylaxis is an important aim of modern medicine. The prophylactic strategies should be based on assigning groups of patients to risk categories. Our result clearly indicates that patients can be divided into 3 risk groups. Administration of low molecular weight heparin or other antithrombus drugs, such as factor Xa inhibitor, are presently recommended for patients with high VTE risk. ${ }^{6}{ }^{13)}$ Antithrombotic agents are routinely used in patients undergoing general or orthopedic surgery and in hospitalized medical patients, but not usually given in patients with brain tumors because of the risk for anticoagulant-associated intracranial bleeding, especially in early postoperative period. ${ }^{11,14,29)}$ To obtain the maximum efficacy with minimum risk, patient selection for anticoagulant indication is highly recommended. The present scoring strategy might be beneficial for this purpose.
Our study has several limitations. This single center study had a relatively small sample size. Moreover, this study examined the minimum number of parameters. More factors, such as heart failure, respiratory failure, and recent trauma, were examined in other reports. ${ }^{5,21)}$ The present study focused on patients with neuroepithelial tumor, which is considered to be a highly specific condition. Our scoring strategy should be considered as a diseasespecific model, which is applicable only to patients with neuroepithelial tumor. This preliminary study was intended only to develop the risk assessment score. A more universal study, such as a multicenter prospective observational study, is recommended to develop a derivation and validation cohort.

The present study demonstrated that the risk of VTE in patients with neuroepithelial tumor can be assessed using a simple risk score model based on 4 parameters. This patient stratification of VTE risk might be useful to identify patients who would benefit from thromboprophylaxis.

\section{Conflicts of Interest Disclosure}

The authors have no personal financial or institutional interest in any of the drugs, materials, or devices in the article. All authors have registered online Self-reported COI Disclosure Statement Forms through the website for JNS members.

\section{References}

1) Agnelli G, Piovella F, Buoncristiani P, Severi P, Pini M, D’Angelo A, Beltrametti C, Damiani M, Andrioli GC, Pugliese R, Iorio A, Brambilla G: Enoxaparin plus compression stockings compared with compression stockings alone in the prevention of venous thromboembolism after elective neurosurgery. $N$ Engl J Med 339: 80-85,1998

2) Athale UH, Chan AK: Thrombosis in children with acute lymphoblastic leukemia. Part II. Pathogenesis of thrombosis in children with acute lymphoblastic leukemia: effects of the disease and therapy. Thromb Res 111: 199-212, 2003

3) Ay C, Dunkler D, Simanek R, Thaler J, Koder S, Marosi C, Zielinski C, Pabinger I: Prediction of venous thromboembolism in patients with cancer by measuring thrombin generation: results from the Vienna Cancer and Thrombosis Study. J Clin Oncol 29: 2099-2103, 2011

4) Ay C, Vormittag R, Dunkler D, Simanek R, Chiriac AL, Drach J, Quehenberger P, Wagner O, Zielinski C, Pabinger I: D-dimer and prothrombin fragment $1+2$ predict venous thromboembolism in patients with cancer: results from the Vienna Cancer and Thrombosis Study. J Clin Oncol 27: 4124-4129, 2009

5) Barbar S, Noventa F, Rossetto V, Ferrari A, Brando- 
lin B, Perlati M, De Bon E, Tormene D, Pagnan A, Prandoni P: A risk assessment model for the identification of hospitalized medical patients at risk for venous thromboembolism: the Padua Prediction Score. J Thromb Haemost 8: 2450-2457, 2010

6) Becattini C, Lignani A, Agnelli G: New oral anticoagulants for venous thromboembolism: focus on factor Xa and thrombin inhibitors. Curr Drug Discov Technol 9: 119-128, 2012

7) Brandes AA, Scelzi E, Salmistraro G, Ermani M, Carollo C, Berti F, Zampieri P, Baiocchi C, Fiorentino MV: Incidence and risk of thromboembolism during treatment of high-grade gliomas: a prospective study. Eur J Cancer 33: 1592-1596, 1997

8) Brown MD, Lau J, Nelson RD, Kline JA: Turbidimetric D-dimer test in the diagnosis of pulmonary embolism: a metaanalysis. Clin Chem 11: 1846-1853, 2003

9) Brown MD, Rowe BH, Reeves MJ, Bermingham JM, Goldhaber SZ: The accuracy of the enzyme-linked immunosorbent assay D-dimer test in the diagnosis of pulmonary embolism: a meta-analysis. Ann Emerg Med 2: 133-144, 2002

10) Chew HK, Wun T, Harvey D, Zhou H, White RH: Incidence of venous thromboembolism and its effect on survival among patients with common cancers. Arch Intern Med 166: 458-464, 2006

11) Dentali F, Douketis J, Gianni M, Lim W, Crowther MA: Metaanalysis: anticoagulant prophylaxis to prevent symptomatic venous thromboembolism in hospitalized medical patients. Ann Intern Med 146: 278-288, 2007

12) Flinn WR, Sandager GP, Silva MB Jr, Benjamin ME, Cerullo LJ, Taylor M: Prospective surveillance for perioperative venous thrombosis: experience in 2643 patients. Arch Surg 131: 472-480, 1996

13) Galanis T, Thomson L, Palladino M, Merli GJ: New oral anticoagulants. J Thromb Thrombolysis 31: 310-320, 2011

14) Geerts WH, Bergqvist D, Pineo GF, Heit JA, Samama CM, Lassen MR, Colwell CW; American College of Chest Physicians: Prevention of venous thromboembolism: American College of Chest Physicians Evidence-Based Clinical Practice Guideline (8th Edition). Chest 133: 381S-453S, 2008

15) Geerts WH, Pineo GF, Heit JA, Bergqvist D, Lassen MR, Colwell CW, Ray JG: Prevention of venous thromboembolism: the Seventh ACCP Conference on Antithrombotic and Thrombolytic Therapy. Chest 126: 338S-400S, 2004

16) Gerber DE, Grossman SA, Streiff MB: Management of venous thromboembolism in patients with primary and metastatic brain tumors. J Clin Oncol 24: 1310-1318, 2006

17) Goldhaber SZ, Grodstein F, Stampfer MJ, Manson JE, Colditz GA, Speizer FE, Willett WC, Hennekens CH: A prospective study of risk factors for pulmonary embolism in women. JAMA 277: 642-645, 1997

18) Goldhaber SZ, Savage DD, Garrison RJ, Castelli WP,
Kannel WB, McNamara PM, Gherardi G, Feinleib M: Risk factors for pulmonary embolism. The Framingham Study. Am J Med 74: 1023-1028, 1983

19) Heinemann LA, Dominh T, Assmann A, Schramm W, Schürmann R, Hilpert J, Spannagl M: VTE Risk assessment-a prognostic Model: BATER Cohort Study of young women. Thromb J 3: 5, 2005

20) Horlander KT, Mannino DM, Leeper KV: Pulmonary embolism mortality in the United States, 1979-1998: an analysis using multiple-cause mortality data. Arch Intern Med 163: 1711-1717, 2003

21) Jenkins EO, Schiff D, Mackman N, Key NS: Venous thromboembolism in malignant gliomas. J Thromb Haemost 8: 221-227, 2010

22) Kakkar VV, Howe CT, Nicolaides AN, Renney JT, Clarke MB: Deep vein thrombosis of the leg: is there a "high risk" group? Am J Surg 120: 527-530, 1970

23) Kawaguchi T, Kumabe T, Kanamori M, Nakamura T, Saito R, Yamashita Y, Sonoda Y, Watanabe M, Tominaga T: Early detection of venous thromboembolism in patients with neuroepithelial tumor: efficacy of screening with serum D-dimer measurements and Doppler ultrasonography. J Neurooncol 101: 495-504, 2011

24) Khorana AA, Francis CW, Culakova E, Kuderer NM, Lyman GH: Frequency, risk factors, and trends for venous thromboembolism among hospitalized cancer patients. Cancer 110: 2339-2346, 2007

25) Khorana AA, Kuderer NM, Culakova E, Lyman GH, Francis CW: Development and validation of a predictive model for chemotherapy-associated thrombosis. Blood 111: 4902-4907, 2008

26) Louis DN, Ohgaki H, Wiestler OD, Cavenee WK: WHO Classification of Tumours of the Central Nervous System, ed 4. Lyon, IARC, 2007

27) Nashef SA, Roques F, Michel P, Gauducheau E, Lemeshow S, Salamon R: European system for cardiac operative risk evaluation (EuroSCORE). Eur J Cardiothorac Surg 16: 9-13, 1999

28) Ozsoylu S, Strauss HS, Diamond LK: Effects of corticosteroids on coagulation of the blood. Nature 195: 1214-1215, 1962

29) Perry JR, Julian JA, Laperriere NJ, Geerts W, Agnelli G, Rogers LR, Malkin MG, Sawaya R, Baker R, Falanga $\mathrm{A}$, Parpia S, Finch T, Levine MN: PRODIGE: a randomized placebo-controlled trial of dalteparin low-molecular-weight heparin thromboprophylaxis in patients with newly diagnosed malignant glioma. J Thromb Haemost 8: 1959-1965, 2010

30) Rogers SO Jr, Kilaru RK, Hosokawa P, Henderson WG, Zinner MJ, Khuri SF: Multivariable predictors of postoperative venous thromboembolic events after general and vascular surgery: results from the patient safety in surgery study. J Am Coll Surg 204: 1211-1221, 2007

31) Roques F, Nashef SA, Michel P, Gauducheau E, de Vincentiis C, Baudet E, Cortina J, David M, Faichney A, Gabrielle F, Gams E, Harjula A, Jones MT, Pintor PP, Salamon R, Thulin L: Risk factors and outcome 
in European cardiac surgery: analysis of the EuroSCORE multinational database of 19030 patients. Eur J Cardiothorac Surg 15: 816-823, 1999

32) Samama MM, Dahl OE, Quinlan DJ, Mismetti P, Rosencher N: Quantification of risk factors for venous thromboembolism: a preliminary study for the development of a risk assessment tool. Haematologica 88: 1410-1421, 2003

33) Semrad TJ, O’Donnell R, Wun T, Chew H, Harvey D, Zhou H, White RH: Epidemiology of venous thromboembolism in 9489 patients with malignant glioma. J Neurosurg 106: 601-608, 2007

34) Spyropoulos AC, Anderson FA Jr, Fitzgerald G, Decousus H, Pini M, Chong BH, Zotz RB, Bergmann JF, Tapson V, Froehlich JB, Monreal M, Merli GJ, Pavanello R, Turpie AG, Nakamura M, Piovella F, Kakkar AK, Spencer FA; IMPROVE Investigators: Predictive and associative models to identify hospitalized medical patients at risk for VTE. Chest 140: 706-714, 2011

35) Stassen PM, Derks RP, Kallenberg CG, Stegeman CA: Venous thromboembolism in ANCA-associated vasculitis-incidence and risk factors. Rheumatology (Oxford) 47: 530-534, 2008

36) Stein PD, Beemath A, Meyers FA, Skaf E, Sanchez J,
Olson RE: Incidence of venous thromboembolism in patients hospitalized with cancer. Am J Med 119: 60-68, 2006

37) Stein PD, Henry JW: Prevalence of acute pulmonary embolism among patients in a general hospital and at autopsy. Chest 108: 978-981, 1995

38) Stein PD, Hull RD, Patel KC, Olson RE, Ghali WA, Brant R, Biel RK, Bharadia V, Kalra NK: D-dimer for the exclusion of acute venous thrombosis and pulmonary embolism: a systematic review. Ann Intern Med 8: 589-602, 2004

39) White RH, Gettner S, Newman JM, Trauner KB, Romano PS: Predictors of rehospitalization for symptomatic venous thromboembolism after total hip arthroplasty. N Engl J Med 343: 1758-1764, 2000

Address reprint requests to: Toshihiro Kumabe, MD, Department of Neurosurgery, Kitasato University School of Medicine, 1-15-1 Kitasato, Minami-ku, Sagamihara 252-0374, Japan. e-mail:kuma@kitasato-u.ac.jp 